\title{
The New Internal Medicine Subinternship Curriculum Guide: a Report from the Alliance for Academic Internal Medicine
}

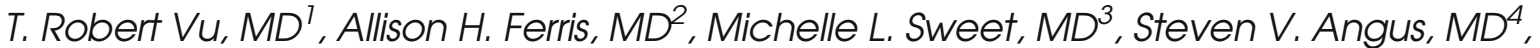 \\ Nadia J. Ismail, $M D^{5}$, Emily Stewart, $M D^{6}$, Jonathan S. Appelbaum, $M D^{7}$, and Brian Kwan, $M D^{8}$
}

${ }^{1}$ Indiana University School of Medicine, Indianapolis, IN, USA; ${ }^{2}$ Charles E. Schmidt College of Medicine, Florida Atlantic University, Boca Raton, FL, USA; ${ }^{3}$ Rush Medical College, Rush University, Chicago, IL, USA; ${ }^{4}$ University of Connecticut School of Medicine, Farmington, CT, USA; ${ }^{5}$ Baylor College of Medicine, Houston, TX, USA; ${ }^{6}$ Sydney Kimmel Medical College, Thomas Jefferson University, Philadelphia, PA, USA; ${ }^{7}$ Florida State University College of Medicine, Tallahassee, FL, USA; ${ }^{8}$ University of California, San Diego, School of Medicine, San Diego, CA, USA.

The internal medicine (IM) subinternship has been a longestablished clinical experience in the final phase of medical school deemed by key stakeholders as a crucial rotation to prepare senior medical students for internship. Medical education has changed greatly since the first national curriculum for this course was developed in 2002 by the Clerkship Directors in Internal Medicine (CDIM). Most notably, competency-based medical education (CBME) has become a fixture in graduate medical education and has gradually expanded into medical school curricula. Still, residency program directors and empirical studies have identified gaps and inconsistencies in knowledge and skills among new interns. Recognizing these gaps, the Association of Program Directors in Internal Medicine (APDIM) surveyed its members in 2010 and identified four core skills essential for intern readiness. The Association of American Medical Colleges (AAMC) also published 13 core entrustable professional activities (EPAs) for entering residency to be expected of all medical school graduates. Results from the APDIM survey along with the widespread adoption of CBME informed this redesign of the IM subinternship curriculum. The authors provide an overview of this new guide developed by the Alliance for Academic Internal Medicine (AAIM) Medical Student-to-Resident Interface Committee (MSRIC).

KEY WORDS: subinternship; acting internship; undergraduate medical education; curriculum.

J Gen Intern Med 34(7):1342-7

DOI: $10.1007 /$ s1 1606-019-04957-0

(C) Society of General Internal Medicine 2019

\section{INTRODUCTION}

The transition from medical student to intern is a significant event along the educational continuum. Interns are expected to assume higher levels of responsibility and increased workloads in challenging clinical, social, and emotional contexts. Yet, gaps and variability in knowledge and skills among new interns have been identified by residency program directors ${ }^{1-3}$ and

Prior Presentations An overview of this new subinternship curriculum was presented in two workshops at the national CDIM conference in San Antonio, Texas, March 20th and 21st, 2018.

Published online April 1, 2019 empirical studies. ${ }^{4-7}$ These gaps have prompted medical educators to focus on the fourth year of medical school as an opportunity to strengthen students' preparedness for internship. ${ }^{2,8-12}$ Among the wide array of fourth-year courses, the subinternship, or acting internship, is commonly viewed by faculty ${ }^{2,12}$ and residents ${ }^{13}$ as one of the most important clinical experiences in helping senior students prepare for internship.

The subinternship's origin dates back to World War II when a national shortage of interns necessitated the creation of "acting internships" for senior medical students. This new rotation subsequently became widely adopted ${ }^{14}$ and is now required at $90 \%$ of US allopathic medical schools. ${ }^{10}$ Despite its longstanding tenure, medical educators only started to standardize structure and content for the subinternship over the past two decades. In 1998, Fagan and colleagues began this process by outlining specific recommendations for the internal medicine (IM) subinternship's structure and experience. ${ }^{15}$ In 2002, the Clerkship Directors in Internal Medicine (CDIM) Subinternship Task Force developed a core curriculum for the IM subinternship ${ }^{16,17}$ derived from a needs assessment survey of IM residency program directors, subinternship directors, and interns. ${ }^{18}$

Since the turn of this century, the landscape of graduate medical education (GME) has shifted significantly with changes in duty hour regulations, increased patient handoffs, adoption of competency-based medical education, and implementation of electronic health records, among others, resulting in a national call for medical school curriculum reform to better equip medical students for post-graduate training. In 2010, the Association of Program Directors in Internal Medicine (APDIM) surveyed its members and proposed four core skills all IM interns should possess at the start of residency. ${ }^{19}$ Soon thereafter, the Alliance for Academic Internal Medicine (AAIM) formed a joint CDIM-APDIM committee to examine the fourth year of medical school with the aim of developing evidence-based recommendations to help students optimize their preparation for internship. ${ }^{10-13,20}$ The Association of American Medical Colleges (AAMC) also established 13 core entrustable professional activities (EPAs) for entering residency, defining a set of foundational skills and behaviors expected of all medical school graduates (Table 1). ${ }^{21,22}$ The core EPAs 
were created as a practical approach to assess the Accreditation Council for Graduate Medical Education (ACGME) six core competencies in workplace settings. Concurrently, the medical education community became increasingly aware of the high degree of distress and burnout among trainees, ${ }^{23-25}$ and made an urgent appeal for interventions to improve medical students' well-being and resilience in hopes of enhancing their preparedness for the next phase of training. ${ }^{26,27}$

In response to the vastly changed GME landscape and calls $^{8-12,19}$ for better preparation of medical graduates, the AAIM Medical Student-to-Resident Interface Committee (MSRIC) created an updated IM subinternship curricular guide for subinternship directors and core teaching faculty. In a parallel process, the AAIM Undergraduate Medical Education (UME) Task Force highlighted the most vital EPAs and foundational skills during the core IM clerkship, which function as important prerequisites to the subinternship. ${ }^{28}$ Redesign of the subinternship curriculum was intended to include developmentally appropriate competencies that could be assessed during this important rotation to ensure readiness for internship. This paper provides an overview of this new curricular guide and describes how it was developed.

\section{CURRICULUM DEVELOPMENT PROCESS}

Building on the previously published IM subinternship curriculum $^{16,17}$ and primer, ${ }^{29}$ key domains from these documents were used to develop survey items for the 2010 APDIM survey. Results from this survey, ${ }^{19}$ recommendations from the joint CDIM-APDIM position paper about the IM subinternship, ${ }^{12}$ and increasing recognition of medical trainee burnout informed MSRIC's work on this project. From the APDIM survey, IM residency program directors identified four core skills expected of new interns: patient evaluation skills to recognize sick vs. non-sick patients, knowing when to ask for assistance, managing time wisely, and communicating

Table 1 The Association of American Medical Colleges (AAMC) 13 Core EPAs

\begin{tabular}{ll}
\hline \hline EPA 1 & $\begin{array}{l}\text { Gather a history and perform a physical examination } \\
\text { Prioritize a differential diagnosis following a clinical } \\
\text { encounter }\end{array}$ \\
EPA 2 & $\begin{array}{l}\text { Recommend and interpret common diagnostic and } \\
\text { screening tests }\end{array}$ \\
EPA 3 & $\begin{array}{l}\text { Enter and discuss orders and prescriptions } \\
\text { Document a clinical encounter in the patient record }\end{array}$ \\
EPA 4 & Provide an oral presentation of a clinical encounter \\
EPA 5 & $\begin{array}{l}\text { Form clinical questions and retrieve evidence to advance } \\
\text { patient care }\end{array}$ \\
EPA 6 & $\begin{array}{l}\text { Give or receive a patient handover to transition care } \\
\text { responsibility }\end{array}$ \\
EPA 8 & $\begin{array}{l}\text { Collaborate as a member of an interprofessional team } \\
\text { Recognize a patient requiring urgent or emergent care } \\
\text { and initiate evaluation and management }\end{array}$ \\
EPA 9 & $\begin{array}{l}\text { Obtain informed consent for tests and/or procedures } \\
\text { EPA 10 }\end{array}$ \\
EPA 11 & $\begin{array}{l}\text { Perform general procedures of a physician } \\
\text { Identify system failures and contribute to a culture of }\end{array}$ \\
EPA 12 & safety and improvement \\
EPA 13
\end{tabular}

Source: https://members.aamc.org/eweb/upload/core\%20EPA\%20Curriculum\%20Dev\%20Guide.pdf. Accessed 29 October 2018 effectively within healthcare teams. ${ }^{19}$ While two of the four core skills map directly to similar core EPAs for entering residency, all directly relate to the core tenet of entrustment, or trustworthiness, central to the EPA concept: trainees must consistently demonstrate conscientiousness, truthfulness, and discernment (knowing their own limits and seeking help). As previously noted, some of the core EPAs for entering residency were regarded as important to assess during the core IM clerkship. ${ }^{28}$ These EPAs, in turn, serve as prerequisites for other core EPAs. For example, the ability to gather a history and perform a physical examination (EPA-1), prioritize a differential diagnosis following a clinical encounter (EPA-2), recommend and interpret common diagnostic/screening tests (EPA-3), and enter and discuss orders/prescriptions (EPA-4) are a group of prerequisite skills needed at a basic level of entrustment before a trainee can recognize a patient requiring urgent/emergent care and initiate appropriate evaluation and management (EPA-10).

To focus on intern readiness, MSRIC chose to use the four core skills outlined by the IM residency program directors along with medical student wellness as the central framework for development of this guide. These five domains have a natural and logically linked relationship with one another especially when viewed in the healthcare workplace context (Fig. 1). In addition to mapping these core skills to the EPAs, we also utilized the RIME (Reporter-Interpreter-Manager-Educator) framework ${ }^{30}$ for its practical linkage to patient care responsibilities and core EPAs, ${ }^{31}$ its developmental nature, ${ }^{32}$ and its predictive validity of future performance during internship. $^{33}$ Table 2 outlines the link between the supporting core EPAs, ACGME competencies, and RIME model to the four core skills recommended by IM program directors.

This guide's learning objectives are written using EPA language to parallel the AAMC's core EPAs. Table 3 summarizes the learning objectives for each of the five core skills of this curriculum. A thorough review of the medical education literature was conducted to provide the most updated and relevant curricular content to support the learning objectives. Users of this guide will note that the core skill of "Knowing When to Ask for Help" also serves as a learning objective under other core skills, while some other learning objectives repeat themselves under different core skills. This redundancy is meant to emphasize the tight interrelationships between these content areas in how one domain enables the other. For example, prioritizing patients' clinical problems according to degree of clinical importance/urgency, a core "Patient Evaluation" skill, is also required to improve one's efficiency and time management.

\section{CURRICULUM CONTENT OVERVIEW}

Although not specifically included in the 13 core EPAs, "time management" is a core skill that may serve as a framework to help subinterns organize and prioritize their professional 


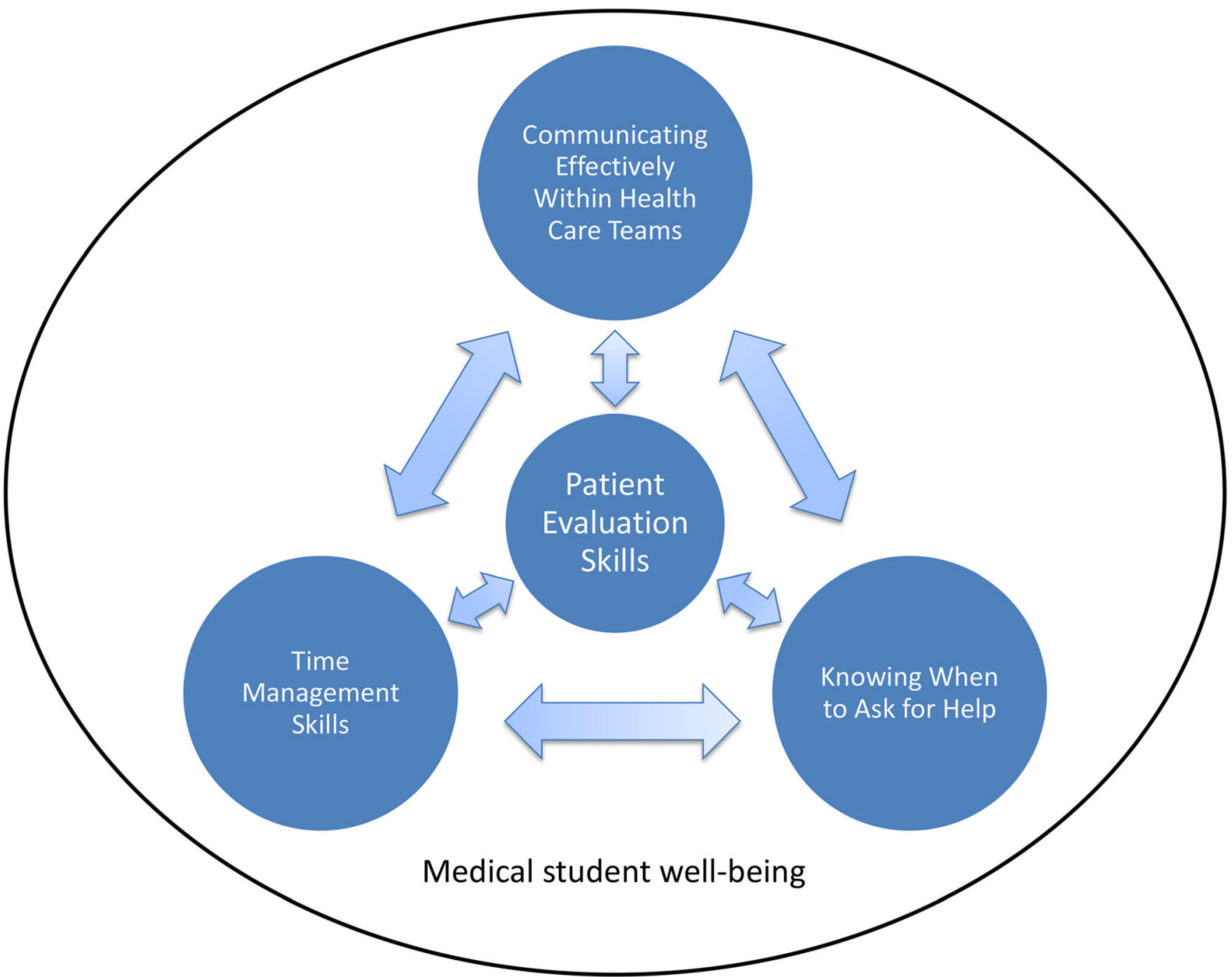

Figure 1 Interrelationships of the five core skills for subinterns.

activities. While third-year clerkships allow trainees to practice and develop clinical skills at a forgiving pace, the subinternship is a more challenging rotation that requires learners to apply these skills in a more timely and practical manner. Subinterns must not only learn how to prioritize different activities throughout the day (e.g., entering orders and calling

Table 2 Relationship Between Core Skills from APDIM Survey, Core EPAs for Entering Residency and Their Prerequisites, Core ACGME Competencies, and RIME Model

\begin{tabular}{lllll}
\hline $\begin{array}{l}\text { Core skills from } \\
\text { APDIM survey }\end{array}$ & $\begin{array}{l}\text { Corresponding core } \\
\text { EPA for entering } \\
\text { residency }\end{array}$ & $\begin{array}{l}\text { Prerequisite/ } \\
\text { enabling, or related } \\
\text { core EPAs }\end{array}$ & $\begin{array}{l}\text { Corresponding } \\
\text { ACGME } \\
\text { competencies }\end{array}$ & RIME model \\
\hline $\begin{array}{l}\text { Time management skills } \\
\text { N/A }\end{array}$ & N/A & PROF, ICS, SBP, PC & $\begin{array}{l}\text { Consistent reporter and interpreter } \\
\text { with some initial manager skills }\end{array}$ \\
$\begin{array}{l}\begin{array}{l}\text { Communicating effectively } \\
\text { within healthcare teams }\end{array} \\
\begin{array}{l}\text { Patient evaluation skills- } \\
\text { recognizing sick } \\
\text { vs. non-sick patients }\end{array}\end{array}$ & EPA-10 & EPA-5, EPA-6, EPA-8 & ICS, SBP, PROF, PC & $\begin{array}{l}\text { Consistent reporter and interpreter } \\
\text { with some initial manager and } \\
\text { educator skills }\end{array}$ \\
$\begin{array}{l}\text { Knowing when to ask } \\
\text { for assistance }\end{array}$ & N/A & EPA-1, EPA-2, EPA-3, EPA-4 & PC, MK, ICS, SBP & $\begin{array}{l}\text { Consistent reporter and interpreter } \\
\text { with some initial manager skills }\end{array}$ \\
\hline
\end{tabular}

PC patient care, MK medical knowledge, ICS interpersonal and communication skills, SBP systems-based practice, PBLI practice-based learning and improvement, PROF professionalism 
Table 3 Subinternship Core Skills and Their Learning Objectives

\begin{tabular}{l}
\hline \hline Subinternship core skill \\
\hline I. Time management skills
\end{tabular}
within healthcare teams

\section{Learning objectives}

1. Organize a daily patient care task list for each patient in a structured and systematic way so that required tasks (e.g., daily notes, orders, etc) are not overlooked.

2. Prioritize daily patient care task list according to degree of importance/urgency.

3. Prioritize patients' clinical problems according to degree of clinical importance/urgency.

4. Recognize one's own limitations and call on other team members to help.

1. Write accurate, concise, and well-organized transfer/accept notes, discharge summaries, and cross-cover notes 2. Provide an oral presentation of a clinical encounter, tailoring length and content according to context

3. Give and receive patient handoffs (both in writing and verbally) to transition care responsibility

4. Speak with specialist/subspecialist colleagues to request consultation

5. Communicate collaboratively with nursing and pharmacy staff to enhance patient care

6. Communicate effectively with team case manager, social worker, and outpatient care providers to facilitate discharge planning

III. Patient evaluation skills-recognizing sick vs. non-sick patients

1. Gather appropriate clinical data from all appropriate sources (e.g., patient, family, nurse, medical records) in hypothesis-driven fashion to address the main clinical problems (Reporter function of RIME)

2. Analyze and synthesize the collected clinical data set to formulate a prioritized differential diagnosis for the main undifferentiated problems (Interpreter function of RIME)

3. Recognize which clinical situations require additional assistance from upper level resident, faculty attending, and/or specialty consultants

4. Develop initial diagnostic and/or therapeutic management plans for the main clinical problems

(Manager function of RIME)

5. Prioritize problem list according to degree of clinical importance (Interpreter function of RIME)

IV. Knowing when to ask for assistance

1. Recognize various techniques that can enhance and develop metacognitive skills

2. Generate clinical questions and retrieve evidence to advance patient care

3. Identify clinical and contextual situations that require assistance from clinical supervisors

4. Utilize a communication framework when calling for clinical support

V. Medical student wellness

1. Utilize a validated tool to assess one's personal risk of burnout

2. Recall multiple evidence-based interventions that may aid in wellness

3. Reflect on identified "at-risk" domains and experiment with suggested interventions to improve wellness

4. Incorporate helpful techniques into daily practice by creating a behavior change plan (BCP) consultations prior to documentation on a sick patient) but also develop a comprehensive method to track incoming data and execute patient-care tasks. This curricular guide includes a sample organizational schema called "the one-page system" (Fig. 2) that subinterns can use to develop their own personalized approach.

The second core skill of "communicating effectively within healthcare teams" stresses the importance of subinterns honing their written and verbal communication skills to cohesively summarize and convey important issues at critical transition points of care. More fundamental documentation skills, such as admission history and physical exams and daily progress notes, are typically acquired during core third-year clerkships. The subinternship curriculum instead focuses on more developmentally appropriate documentation dealing with transitions of care, important junctures that can leave patients particularly vulnerable. Accordingly, transfer/accept notes, discharge summaries, cross-cover notes, and handoffs are the main focus of this guide. Details of interdisciplinary teamwork and communication-including communicating with consultants - to facilitate patient care are also described.

For the "patient evaluation" core skill, a representative list of common medical emergencies was chosen to serve as a guide and not intended to be an exhaustive list. MSRIC chose not to develop actual cases for these problems but rather provide a structured guide that subinternship directors and teaching faculty can use to tailor their own teaching cases according to their institution's individualized needs. The format for each problem was adapted from Lange's Internal Medicine On Call (with permission from the authors) for its undifferentiated problembased approach and stepwise thought process that occurs during the cross-coverage experience to help subinterns develop their patient evaluation skills. ${ }^{34}$

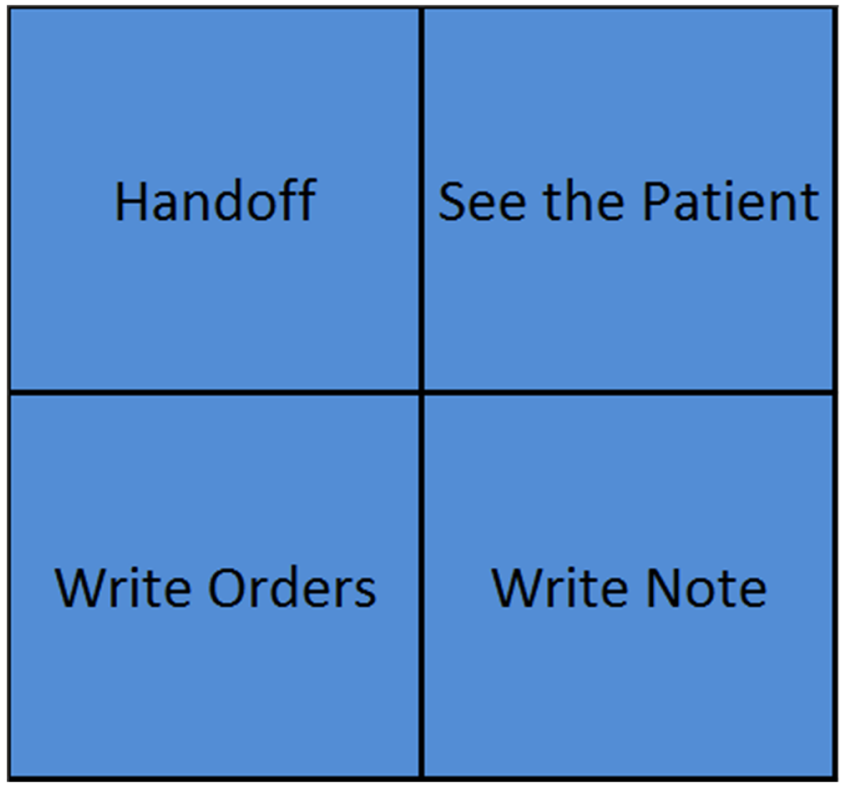

Figure 2 The one-page box system: a visual aid developed to guide daily workflow and assist with student organizational skills. The complete description of this system can be found in the full curricular guide. 
As a trainee, the core skill of "asking for help" can be challenging when navigating the tension between preserving autonomy and professional credibility while maintaining patient safety. Recognizing that the process of requesting assistance is multi-faceted and complex, ${ }^{35}$ MSRIC chose to focus on factors related to trainee development. Specifically, the curriculum describes various metacognitive techniques that can improve clinical reasoning and stresses the importance of self-assessment for the purpose of practice improvement. ${ }^{36}$ It also introduces the PICO model ${ }^{37}$ (Patient/problem/population, Intervention, Comparison/control/comparator, Outcome) to build well-structured clinical questions. Lastly, a widely used and practical communication tool, $\mathrm{SBAR}^{38}$ (Situation, Background, Assessment, Recommendation), is provided to help students structure their communication more effectively when asking for help or giving handoffs. Together, these resources can enable trainees to recognize what they do not know, develop a means to answer those questions in an evidence-based manner, and query their supervisors in a systematic fashion.

The final component of the curriculum is a focus on wellness and self-care. This section includes an overview of the existing literature on burnout pertaining to medical students, provides a validated tool to assist with self-assessment of burnout risk, ${ }^{39}$ and reviews evidence-based interventions that can aid wellness, including development of a "behavioral change plan" 40 which can help learners implement self-care into their daily lives.

\section{CHALLENGES AND FUTURE DIRECTIONS}

The goal of this curriculum is to emphasize important, developmentally appropriate skills for the final phase of medical schools' curricula to help trainees' readiness for internship. However, barriers exist to prevent a cohesive implementation plan that would allow a single fit for all institutions. Each medical school will need to work within the limits of their existing curriculum and assessment methods, which will undoubtedly cause wide variations in implementation of content as well as methodology.

Additionally, it may be ambitious to implement this entire curriculum within the confines of a traditional four-week IM subinternship. Although derived from a survey of IM program directors, one could argue that this curriculum's proposed skills are broadly applicable to all graduating students. Many topics in this curriculum can be adopted by other fourth-year courses of medical school to reinforce important concepts and may especially be relevant for a capstone or "boot camp" preparatory course prior to internship.

Although this curriculum is linked with the AAMC EPAs, not all medical schools have fully adopted EPAs. Schools that have adopted EPA-based curricula are still piloting different assessment methods for the EPAs (i.e., levels of entrustability) at the UME level, ${ }^{41,}{ }^{42}$ so validated tools for any EPA-based assessment are not yet available. However, some sample evaluation tools are included in the Appendix section of this curricular guide.

Finally, some of the core skills of this new curriculum are considered "life skills." In particular, time management, selfawareness to know one's limits, and stress/burnout management are challenging topics to incorporate into any curricula. These topics are ideal for self-assessment, but all three, especially wellness, may prove to be challenging to incorporate into traditional faculty-driven assessment models. Additionally, these topics may not be adequately incorporated or assessed if condensed into a finite block of time.

Further work is needed to develop validated EPA-based assessment tools for this new curriculum and to gather empirical data on its effectiveness in preparing students for internship. Pilot testing at several institutions is underway to focus on these two goals.

\section{CONCLUSION}

The IM subinternship remains a critically important clinical experience for medical students to help their transition into post-graduate training. This new subinternship curricular guide is a practical, evidence-based toolkit to enhance intern readiness, integrating IM program directors' perspectives to emphasize core skills and EPAs most relevant to IM residency. $^{43}$

\section{Acknowledgements:}

The authors would like to acknowledge the Alliance for Academic Internal Medicine staff, especially Christopher M. Williams, MPH, in their support of the Medical Student-to-Resident Interface Committee's work on this project. The authors also acknowledge Cassandra Laughlin for her assistance with manuscript preparation.

Contributors: The Clerkship Directors in Internal Medicine (CDIM) Council and the Association of Program Directors in Internal Medicine (APDIM) Council reviewed and approved the new subinternship curriculum guide.

Corresponding Author: T. Robert Vu, MD; Indiana University School of Medicine, 1120 West Michigan St., Gatch Hall CL630, Indianapolis, IN, USA (e-mail: tvu@iu.edu).

\section{Compliance with Ethical Standards:}

Conflict of Interest: Two authors disclosed the following potential conflicts of interest: $\mathrm{Dr}$. Vu received an internal grant at his academic institution in 2017, and Dr. Appelbaum served as a consultant for two healthcare-related companies and received an honorarium from another healthcare-related company. The authors note that these relationships with the disclosed entities did not affect this project. All remaining authors declare that they do not have a conflict of interest.

\section{REFERENCES}

1. Langdale LA, Shaad D, Wipf $\mathbf{J}$, et al. Preparing graduates for the first year of residency: Are medical schools meeting the need? Acad Med 2003; 78:39-44.

2. Lyss-Lerman P, Teherani A, Aagaard E, et al. What training is needed in the fourth year of medical school? Views of residency program directors. Acad Med 2009;84:823-829. 
3. Angus SV, Vu TR, Willett LL, Call S, Halvorsen AJ, Chaudhry S Internal medicine residency program directors' views of the core entrustable professional activities for entering residency: An opportunity to enhance communication of competency along the continuum. Acad Med 2017;92(6):785-791.

4. Lypson ML, Frohna JG, Gruppen LD, Woolliscroft JO. Assessing residents' competencies at baseline: identifying the gaps. Acad Med 2004; 79(6):564-570.

5. Lypson ML, Hamstra SJ, Colletti L. M. Using OSCEs to teach WHO patient safety solutions. Med Educ 2008; 42:523-524

6. Lypson ML, Gosbee JW, Andreatta P. Assessing the patient safety knowledge and experiences of trainees. Med Educ 2008; 52:11331134.

7. Wagner D, Lypson ML. Centralized assessment in graduate medical education: cents and sensibilities. J Grad Med Educ 2009; 1(1):21-27.

8. Walling A, Merando A. The fourth year of medical education: A literature review. Acad Med 2010; 85:1698-1704.

9. Reddy ST, Chao J, Carter JL, et al. Alliance for Clinical Education Perspective Paper: Recommendations for redesigning the "Final Year" of medical school. Teach Learn Med 2014; 26(4):420-427.

10. Elnicki DM, Gallagher SJ, Willett $\mathbf{L R}$, et al. for the CACTI Group (CDIM-APDIM Committee on Transitions to Internship). Course Offerings in the Fourth Year of Medical School: How U.S. Medical Schools Are Preparing Students for Internship. Acad Med 2015; 90(10): 1324-1330.

11. Elnicki DM, Aiyer MK, Cannarozzi ML, et al. An Entrustable Professional Activity (EPA)-Based Framework to Prepare Fourth-Year Medical Students for Internal Medicine Careers. J Gen Intern Med 2017; 32(11): 1255-1260.

12. Vu TR, Angus Sv, Aronowitz PB, et al. for the CACTI Group (CDIMAPDIM Committee on Transitions to Internship). The Internal Medicine Subinternship - More Important Now Than Ever: A Joint CDIM-APDIM Position Paper. J Gen Intern Med 2015;30(9):1369-75.

13. Pereira AG, Harrell HE, Weissman A, Smith CD, Dupras D, Kane GC. Important skills for internship and the fourth-year medical school courses to acquire them: a national survey of internal medicine residents. Acad Med 2016; 91:821-826.

14. Ludmerer K. Time to Heal: American Medical Education from the Turn of the Century to the Era of Managed Care. New York: Oxford University Press; 1999.

15. Fagan MJ, Curry RH, Gallagher SJ. The evolving role of the acting internship in the medical school curriculum. Am J Med 1998; 104:40912.

16. Green EH, Fagan MJ, Reddy S, Sidlow R, Mechaber AJ. Advances in the internal medicine subinternship. Am J Med 2002;113:769-773.

17. CDIM Subinternship Curriculum and Training Problems. Available from: https://www.im.org/resources/ume-gme-program-resources/curriculum. Accessed 29 October 2018.

18. Sidlow R, Mechaber AJ, Reddy S, Fagan M, Marantz PR. The internal medicine subinternship: a curriculum needs assessment. J Gen Intern Med 2002; 17:561-564.

19. Angus S, Vu TR, Halvorsen AJ, et al. What skills should new internal medicine interns have in July? A national survey of internal medicine residency program directors. Acad Med 2014; 89:432-435.

20. Chretien KC, Elnicki DM, Levine D, Aiyer M, Steinmann A, willett LR. What are we telling our students? A national survey of clerkship directors' advice for students applying to internal medicine residency. J Grad Med Educ 2015; 7(3):382-387.

21. Core Entrustable Professional Activities for Entering Residency. Available from: www.mededportal.org/icollaborative/resource/887. Accessed 29 October 2018.

22. Englander R, Flynn T, Call S, et al. Toward Defining the Foundation of the MD Degree: Core Entrustable Professional Activities for Entering Residency. Acad Med. 2016;91:1352-1358.
23. Dyrbye LN and Shanafelt TD. A narrative review on burnout experienced by medical students and residents. Med Ed 2016; 50:132-49.

24. Ishak W, Nikravesh R, Lederer S, Perry R, Ogunyemi D, Bernstein C. Burnout in medical students: a systematic review. Clin Teach 2013; 10 : 242-245.

25. Rotstein LS, Ramos MA, Torre M. Prevalence of Depression, Depressive Symptoms, and Suicidal Ideation Among Medical Students: A Systematic Review and Meta-Analysis. JAMA 2016;316(21):2214-2236.

26. Dyrbye LN and Shanafelt TD. Commentary: medical student distress: a call to action. Acad Med 2011; 86(7):801-803.

27. Slavin SJ. Medical Student Mental Health: Culture, Environment and the Need for a Change. JAMA 2016:316(21):2195-2196.

28. Fazio SB, Ledford CH, Aronowitz PB, et al. Competency-Based Medical Education in the Internal Medicine Clerkship: A Report From the Alliance for Academic Internal Medicine Undergraduate Medical Education Task Force. Acad Med 2018; 93:421-427.

29. Harrell H, Aiyer M, Appel J, Gliatto P, Sweet M. Primer to the Internal Medicine Subinternship. MedEdPORTAL; 2012. Available from: www. mededportal.org/publication/9277. Accessed 29 October 2018.

30. Pangaro L. A new vocabulary and other innovations for improving descriptive in-training evaluations. Acad Med 1999;74(11):1203-6.

31. Meyer EG, Kelly WF, Hemmer PA, Pangaro LN. The RIME Model Provides a Context for Entrustable Professional Activities Across Undergraduate Medical Education. Acad Med. 2018;93:954.

32. Santen SA, Seidelman JL, Miller CS, et al. Milestones for Internal Medicine Sub-interns. Am J Med 2015 Jul;128(7):790-8.

33. Lavin B, Pangaro L. Internship ratings as a validity outcome measure for an evaluation system to identify inadequate clerkship performance. Acad Med 1998; 73:998-1002.

34. Haist SA, Robbins JB, Gomella LG. Internal Medicine On Call, Fourth Edition. Lange Medical Books. McGraw-Hill. 2005.

35. Kennedy TJ, Regehr G, Baker GR, Lingard L. Preserving professional credibility: grounded theory study of medical trainees' requests for clinical support. BMJ 2009 Feb 9;338:b128.

36. Colbert CY, Graham L, West $\mathbf{C}$, et al. Teaching metacognitive skills: helping your physician trainees in the quest to "know what they don't know.". Am J Med 2015 Mar; 128(3):318-24.

37. Richardson WS, Wilson MC, Nishikawa J, Hayward RS. The well-built clinical question: a key to evidence-based decisions. ACP J Club 1995 Nov-Dec; 123(3):A12.

38. https://innovations.ahrq.gov/qualitytools/sbar-technique-communication-situational-briefing-model. Accessed 29 October 2018.

39. Dyrbye LN, Schwartz A, Downing SM, Szydlo DW, Sloan JA, Shanafelt TD. Efficacy of a brief screening tool to identify medical students in distress. Acad Med 2011; 86:907-914.

40. Kushner RF, Kessler S, McGaghie WC. Using behavior change plans to improve medical student selfcare. Acad Med 2011;86(7):901-906.

41. Lomis K, Amiel JM, Ryan MS, et al. Implementing an Entrustable Professional Activities Framework in Undergraduate Medical Education: Early Lessons From the AAMC Core Entrustable Professional Activities for Entering Residency Pilot. Acad Med 2017 Jun;92(6):765-770.

42. Brown DR, Warren JB, Hyderi A, et al. Finding a Path to Entrustment in Undergraduate Medical Education: A Progress Report From the AAMC Core Entrustable Professional Activities for Entering Residency Entrustment Concept Group. Acad Med 2017 Jun;92(6):774-779.

43. AAIM Internal Medicine Subinternship Curriculum 2.0. Available from: https://www.im.org/resources/ume-gme-program-resources/curriculum. Accessed 29 October 2018

Publisher's Note Springer Nature remains neutral with regard to jurisdictional claims in published maps and institutional affiliations. 